einzelnen Parlamenten bisher nur unzureichend genutzt werden. Die von ihnen durch eine vermehrte virtuelle Präsenz erwartete Stärkung der demokratischen Legitimation der europäischen Politikgestaltung fällt, angesichts bekannter Repräsentativitätsprobleme und einer sich von anderen Institutionen und Akteuren durchaus unterscheidenden Parlamentsinstanz, jedoch allzu positiv aus.

Es bleibt festzuhalten, dass dieser informative Tagungsband umfangreich und vielseitig die Thematik des Parlamentarismus im EU-Mehrebenensystem erörtert und eine Vielzahl an neuen Erkenntnissen liefert. Die größte Herausforderung liegt nach wie vor in der umfassenden und systematischen empirischen Anwendung der vorliegenden theoretischen Konzepte, insbesondere des Mehrebenenparlamentarismus.

Somit bieten beide Bücher einen guten Überblick über den Stand der Parlamentarismusforschung in der EU. Während der Vorteil der Publikation von Dittgen in der kompakten Deskription der rechtlichen Situation nationaler Parlamente nach Inkrafttreten des Vertrags von Lissabon liegt, ist der Tagungsband von Abels und Eppler naturgemäß deutlich komplexer. Insbesondere die Beiträge der Praktiker (vor allem Preising und Vollrath) überzeugen durch ihr „Insider-Wissen“ zur politischen Praxis der verfassungsrechtlichen Neuerungen. Nun müssen weitere, insbesondere auch ländervergleichende Analysen erfolgen, die über deskriptive Fallstudien hinausgehen.

Ellen Schneider

\title{
Antworten in der Rückschau, Fragen für die Zukunft der europäischen Integration
}

Brincker, Gesa-Stefanie, Mathias Jopp und Lenka Anna Rovná (Hrsg.): Leitbilder for the Future of the European Union. Dissenting Promotors of Unity (Reihe Europäische Schriften, Band 90), Nomos Verlagsgesellschaft, Baden-Baden 2011, 420 Seiten, €69,-.

Stetter, Stephan, Carlo Masala und Marina Karbowski (Hrsg.): Was die EU im Innersten zusammenhält. Debatten zur Legitimität und Effektivität supranationalen Regierens (ZIB-Reader 01), Nomos Verlagsgesellschaft, Baden-Baden 2011, 374 Seiten, € 39,-.

Wie wird Europa in zwanzig oder dreißig Jahren aussehen? Diese „Gretchenfrage“ der europäischen Integration stellte Bundeskanzlerin Angela Merkel in ihrer Rede an der Prager Karls-Universität im April 2012. ${ }^{1}$ Die beiden vorliegenden Sammelbände nehmen sich der anspruchsvollen Aufgabe an, aus der Rückschau auf die politischen Entwicklungen der PostMaastricht-Ära ebenjene Frage nach den Perspektiven und Herausforderungen der europäischen Integration am Anfang des 21. Jahrhunderts näher zu beleuchten. Während der Fokus des Bandes von Gesa-Stefanie Brincker, Mathias Jopp und Lenka Anna Rovná dabei auf der Analyse des Einflusses europapolitischer Leitbilder ,as a key factor in the development of

1 Vgl. Angela Merkel, Rede bei der Diskussionsveranstaltung „Die künftige Gestalt Europas“ an der Juristischen Fakultät der Karls-Universität Prag am 3. April 2012, http://www.bundesregierung. de/Content/DE/Rede/2012/04/2012-04-03-merkel-prag.html (Abruf am 19. April 2012). 
the European Union“ liegt (S. 13), sammeln Stephan Stetter, Carlo Masala und Marina Karbowski zentrale Beiträge der deutschen Integrationsforschung zu Fragen der Legitimität und Effektivität supranationalen Regierens in der EU.

Im Zentrum des aus einem internationalen Forschungsprojekt am Institut für europäische Politik hervorgegangenen Sammelbandes von Brincker, Jopp und Rovná steht die Frage nach dem Einfluss der EU-Osterweiterung auf die europapolitischen Leitbilder und Zielvorstellungen der EU-Mitgliedstaaten. In ihrer Einleitung skizziert Rovná, dass mit dem Ende des Ost-West-Konfliktes fundamentale konzeptionelle, institutionelle und geopolitische Veränderungen einhergingen, die die Herausbildung einer „multitude of Leitbilder“ über die Zukunft der europäischen Integration mit sich brachten (S. 21 f.).

Claudia Ehmke stellt das Forschungsdesign der Länderstudien vor. In Anlehnung an Heinrich Schneider werden Leitbilder als „visions of a desirable future situation “ beziehungsweise als „mental frameworks“ konzeptionalisiert (S. 34). Die Politische Kultur und die institutionellen Interaktionsbeziehungen im EU-System bilden die unabhängige sowie intervenierende Variable; die klassischen Integrationsansätze des Intergouvernementalismus und Supranationalismus als „most valuable starting points“ bilden den konzeptionellen Rahmen (S. 39). Darauf aufbauend werden die Thesen der Fragmentierung, Kontinuität und Reformulierung europapolitischer Leitbilder als Ausgangspunkt für die Evaluation der Leitbildentwicklungen in den „neuen“ und „alten“ Mitgliedstaaten herangezogen.

Tobias Kuhnstein und Claudia Ehmke zeichnen in ihrem Beitrag die allgemeine Entwicklung des politischen Systems der EU im Zeitraum von 2000 (Nizza) bis zur Unterzeichnung des Lissabon-Vertrages (2007) nach. Den Autoren zufolge zeigt sich, dass die EU-Osterweiterung (,widening“) nahezu zwangsläufig eine Vertiefung der politischen Integration („deepening") mit sich brachte (vgl. S. 69 f.). Eine Konvergenz europäischer Finalitätskonzepte sei damit jedoch nicht einhergegangen, so dass sich supranationale und intergouvernementale Leitbilder weiterhin als kontradiktorische Paradigmen gegenüberstünden.

Diese Feststellungen stützen auch die Erkenntnisse der Länderstudien. Sie machen deutlich, dass es nicht ein national dominierendes europapolitisches Leitbild gibt, sondern diese je nach Politikfeld, situativem Kontext und innergesellschaftlicher Akteurskonstellation variieren. Während die Beiträge zu den „alten“ Mitgliedstaaten Deutschland (Gesa-Stefanie Brincker / Max Conzemius) Frankreich (Gesa-Stefanie Brincker / Anne Schmidt) und Großbritannien (Claudia Ehmke) den umfangreichen Forschungsstand der politisch-historischen Pfadabhängigkeiten der jeweiligen nationalen Leitbilder ergänzend aufbereiten, schließen die Länderstudien zu den „neuen“ EU-Mitgliedern eine bisher bestehende Forschungslücke. So zeigen Ivo Šlosarčik, Zuzana Kasáková, Jan Váška und Thomáš Weiss, dass der Beitritt zur EU in Tschechien weder zu einer Revolution der Politischen Kultur noch zu einem signifikanten Anstieg der EU-Aversion geführt hat. Die jeweilige Wirkmächtigkeit der konkurrierenden Leitbilder des „traditionalist-sovereignist“, „,selective realist“ und „community method driven“ (S. 110) basiere vielmehr auf den aktuellen innenpolitischen Machtgemengelagen. Attila Ágh stellt eine „cognitive dissonance“ (S. 261) Ungarns in Bezug auf die Formulierung europäischer Integrationsleitbilder fest. So seien die Ungarn zwar überzeugte EU-Enthusiasten, jedoch führten die hohen Erwartungen an die EU und die anhaltende „domestic malai-

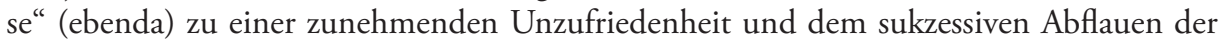
einstmaligen EU-Euphorie. Eine Dissonanz des europapolitischen Selbstverständnisses erkennen auch Marek A. Chichocki und Karolina Czerwińska im Falle Polens. Das historisch bedingte Insistieren auf der nationalen Unabhängigkeit führe zu einer Präferenz des inter- 
gouvernementalen Leitbildes einer „community of nation states“ (S. 286). Die Polen stünden der europäischen Integration dennoch nicht grundsätzlich skeptisch gegenüber. Gerade im Bereich der policy-Leitbilder zeige sich, dass die polnische Position oft zwischen Integrationsskeptizismus und Integrationsbereitschaft changiere.

Folgerichtig kommt Hartmut Marhold in seinem Resümee zu der Einsicht, dass die EUbezogene Leitbildforschung verstärkt die policy-Dimension in den Blick nehmen muss. „History matters“ lautet die zentrale Erkenntnis von Marhold, der den „turn to policies“ als Folgeerscheinung einer Dekade erschöpfender Debatten über die institutionelle Ausgestaltung der EU charakterisiert (vgl. S. 406). Jopp diskutiert in seiner Konklusion die übergreifenden Erkenntnisse aus den Länderstudien und kommt zu dem Fazit, dass man für alle drei Thesen (Fragmentierung, Kontinuität, Reformulierung) Anhaltspunkte fände, jedoch keine vollständig verifiziert werden könne. Dafür ließen sich eine Vergemeinschaftung der national geprägten europapolitischen Konzepte und deren Transformation in „transnational Leitbilder" (S. 418) feststellen.

Der Einfluss europapolitischer Leitbilder und transnationaler Diskurse auf die Organisations- und Interaktionsstrukturen der EU wird auch in dem Sammelband von Stetter, Masala und Karbowski thematisiert. Basierend auf einer postmodernen Analyseperspektive unternimmt Thomas Diez den Versuch, das in Deutschland dominante Integrationsleitbild des europäischen Bundesstaates anhand der Bundestagsdebatten zum Vertrag von Maastricht zu dekonstruieren, um bisher marginalisierte Alternativkonzepte „zum modernen Staat als post-moderne Rekonstruktion“ (S. 51) aufzuzeigen. Andreas Wimmel definiert (S. 107 ff.) transnationale Diskurse als interaktive Kommunikationsstrukturen zwischen verschiedenen (supra-)nationalen Akteuren und verdeutlicht anhand der Debatten über einen EU-Beitritt der Türkei in Deutschland, Frankreich und Großbritannien die fortschreitende Konstituierung einer europäischen Medienöffentlichkeit. Gleichwohl werde diese bisher durch die einseitige Bezugnahme auf (supra-)nationale politische Sprecher dominiert.

Beide Artikel finden sich im ersten Teil des Sammelbandes wieder, in dem sich die Autoren mit den Herausforderungen supranationalen Regierens auseinandersetzen, während sich der zweite Teil den politischen Praktiken in der EU widmet. Insgesamt enthält der als Reader konzipierte Band eine Auswahl von zwölf Beiträgen der deutschsprachigen Europaforschung zu Fragen der Legitimität und Effektivität in der EU, die in den letzten 16 Jahren in der Zeitschrift für Internationale Beziehungen (ZIB) erschienen sind. Bereits in der Einleitung stellen Stetter, Masala und Karbowski darauf ab, dass es angeraten sei, „[...] über den Tellerrand einer konzeptionell auf die EU verengten Integrationsforschung zu blicken“ (S. 7). Die Analysen zur Relevanz und den Problemen supranationalen Regierens in der EU sollten sich intensiver als bisher „an den in den IB schon weit entwickelten Debatten zu Legitimität, Effektivität und Demokratie jenseits des Nationalstaates orientieren“ (S. 6).

Ausgehend von der Überlegung, dass das EU-Mehrebenensystem auf einem heterarchischen Organisationsprinzip basiert, „in dem die politische Autorität zwischen einem Regelungsautor und einem oder mehreren Regelungsadressaten geteilt ist" (S. 25), illustriert Jürgen Neyer anhand verschiedener Politikfelder den Einfluss diskursbasierter Verrechtlichungs- und Vergesellschaftungsprozesse auf die Herrschaftsstrukturen in der EU. Hubert Heinelt legt dar, dass es nötig ist, die theoretische Perspektive auf das europäische Demokratiedefizit zu präzisieren. Eine legitime, effektive und effiziente Politik jenseits der Beschränktheit sektoraler Interessenvermittlung lasse sich demnach durch eine „auf choice- 
Offerten setzende Steuerung als auch durch [...] auf voice orientierte Beteiligungsformen “ (S. 101) erzeugen. Dass sich diese theoretischen Annahmen in der politischen Praxis widerspiegeln, verdeutlichen die Beiträge von Dawid Friedrich (S. 129 ff.) und Frank Wendler (S. 157 ff.), die die Implementation zivilgesellschaftlicher Partizipationsformen im Bereich der europäischen Migrations- und Umweltpolitik beziehungsweise der EU-Sozialpolitik behandeln.

Der zweite Themenkomplex beginnt mit drei Beiträgen zu den Auswirkungen der Verrechtlichung europäischer Politik. Tanja Börzel, Tobias Hofmann und Carina Sprungk nehmen Bezug auf Michael Zürns These zur differenzierten Implementationslogik negativer und positiver Integration und zeigen anhand der Auswertung von 5.500 Rechtsverstößen, dass es „keinen eindeutigen Zusammenhang zwischen dem positiven oder negativen Charakter einer Regelung“ (S. 213) gibt. Dass vielmehr „policy-bezogene Kontextbedingungen“ und nicht „staatsbezogene Variablen wie wirtschaftliche oder politische Macht" entscheidend für den Erfolg von Rechtsdiskursen und Urteilen gegen nationale Rechtsverstöße sind (S. 249 f.), dokumentiert Diana Panke. Die „nationale Bedingtheit der Folgen der europäischen Integration" wird von Susanne K. Schmidt aufgegriffen und am Beispiel der Reformen des deutschen Versicherungswesens und Straßengütersystems nachvollzogen (S. 255 ff.).

Den multiplen Wirkungsbeziehungen zwischen Nationalstaat und EU widmen sich die letzten drei Beiträge des Sammelbandes. So erörtert Antje Wiener am Beispiel der Unionsbürgerschaft „unbeabsichtigte Konsequenzen von Institutionenbildung [...] jenseits des Nationalstaates“ (S. 283), die durch „assoziative Faktoren“ wie Verfassungsnormen und Identitäten hervorgerufen werden und auf die Politik der EU zurückwirken. Dirk Leuffen untersucht anhand der französischen Kohabitation den Einfluss innenpolitischer Variablen auf die Formulierung europäischer Politik und kommt zu dem Fazit: „Domestic politics matters“ (S. 338). Michèle Knodt veranschaulicht, dass auch die EU von anderen Institutionen im weltpolitischen System beeinflusst wird. Ihr Schlussbeitrag skizziert den Wandel europäischer Regierungsroutinen und Legitimitätskonzepte am Beispiel der internationalen Einbettung der EU in die WTO (S. 343 ff.).

Beide Sammelbände zeichnen sich durch ihren stringenten Aufbau und den klar umrissenen konzeptionellen Rahmen aus. Das zentrale Verdienst von Brincker, Jopp und Rovná ist die Einhegung der detailreichen Länderstudien in ein übergeordnetes Forschungsdesign, das die Vergleichbarkeit der nationalen Leitbilder und deren Rückkopplung an den europäischen Integrationsprozess ermöglicht. Inwieweit sich die Konzeptionalisierung der EU-Institutionen als intervenierende Variable nicht als zu reduktionistisch erweist, muss an dieser Stelle offen bleiben. Zumindest sei angemerkt, dass die Deutungs- und Definitionshoheit supranationaler EU-Organe auch in traditionell intergouvernementalen Politikbereichen wie der Bildungspolitik (Bologna-Prozess) beträchtlich sein kann. Ebenso stellt sich die Frage, wieso kein süd- oder nordeuropäisches Land Eingang in die Länderauswahl gefunden hat. Unabhängig davon fallen die zahlreichen - bei einem Preis von 69 Euro besonders - inakzeptablen Mängel in puncto Orthographie und Interpunktion negativ ins Gewicht. Hier kann der Band von Stetter, Masala und Karbowski als Vorbild dienen, der neben der hohen editorischen Qualität mit einer vom jeweiligen Autor kommentierten und dem Artikel vorangestellten Bibliographie aktueller Publikationen zur Beitragsthematik aufwartet. Inhaltlich erweist sich die Verknüpfung der europäischen Integrationsforschung mit konzeptionellen Ansätzen aus den Internationalen Beziehungen sowie die Berücksichtigung empirischer Studien zur Praxis supranationalen Regierens in der EU als große Stärke. 
Obwohl Mitte 2011 publiziert, lassen beide Herausgeberkollektive die integrationspolitische Zäsur im Zeichen der europäischen Staatsschuldenkrise nahezu unkommentiert - ein Wermutstropfen, da gerade konkrete Analysen zu den wirtschafts- und finanzpolitischen Integrationsleitbildern sowie zur Legitimität und Effektivität der EU-Rettungsmechanismen aktuell wichtige Forschungsdesiderate darstellen.

Torben Fischer

Fülle von Einsichten in die deutsch-französischen Beziehungen

Defrance, Corine und Ulrich Pfeil: Deutsch-Französische Geschichte Band 10. Eine Nachkriegsgeschichte in Europa 1945 bis 1963, Wissenschaftliche Buchgesellschaft, Darmstadt 2011, 324 Seiten, € 69,90.

Miard-Delacroix, Hélène: Deutsch-Französische Geschichte Band 11. Im Zeichen der europäischen Einigung 1963 bis in die Gegenwart, Wissenschaftliche Buchgesellschaft, Darmstadt 2011, 404 Seiten, $€ 69,90$.

Lequesne, Christian: Frankreich im neuen Europa (Reihe Denkart Europa. Schriften zur europäischen Politik, Wirtschaft und Kultur, Band 17), Nomos Verlagsgesellschaft, Baden-Baden 2011, 114 Seiten, $€ 15,-$.

Jüngere Darstellungen über die deutsch-französische Nachkriegsgeschichte sind beiderseits des Rheins eher selten. Insofern verdienen die hier anzuzeigenden Bände der elfteiligen Reihe über die beiderstaatliche Geschichte besondere Aufmerksamkeit. Beide füllen eine Lücke - dieses Ergebnis darf schon einleitend festgehalten werden. In ihrer Darstellung der ersten Nachkriegsjahrzehnte geben Corince Defrance, Historikerin an der Universität ParisSorbonne, und Ulrich Pfeil, Professor für Deutschlandstudien an der Universität Metz, zunächst einen etwa 140-seitigen Überblick über die Entwicklung der Nachkriegsbeziehungen. Daran schließt sich ein über 100-seitiger Teil mit „Fragen und Perspektiven“ an. Im ersten Teil ihres Buches geht es zunächst um die Vorstellungen der vierten Besatzungsmacht in Deutschland: „Que faire de l'Allemagne?“ (S. 43). Die verschiedenen schon von den Résistance- und Exilgruppen ausgearbeiteten Pläne sowie die Überlegungen des Chefs der Provisorischen Regierung nach der Befreiung Frankreichs im Sommer 1944, General Charles de Gaulle, werden vorgestellt und gewertet. Dabei bemühen sich die Autoren unter Berücksichtigung neuester Forschungsergebnisse, die französische Besatzungspolitik differenzierter darzustellen, als dies lange Zeit geschah. „Die Deutschlandpolitik de Gaulles [...] rankte sich [zunächst] um die drei Pole: Rang [Frankreichs in der Welt], Sicherheit und Reparationen (besonders Kohle)“ (S. 46). In diese Periode fallen die Erwägungen, das damalige Reichsgebiet eher zu dezentralisieren als auseinander zu brechen. De Gaulles vordringlichstes Ziel war, Einfluss auf das in der britischen Besatzungszone gelegene Ruhrgebiet zu gewinnen. „Aber weder die USA noch Großbritannien noch die Sowjetunion ließen sich zu einer Abtrennung von Rheinland und Ruhrgebiet bewegen“ (S. 50): Die französischen Maximalforderungen, die auch nach dem Rücktritt des Generals im Januar 1946 von dessen Nachfolgern erhoben wurden, hatten folglich keine Chance. Im Gegenteil: vor dem sich zunehmend schärfer 